\title{
Amlexanox enhances temozolomide-induced anti- tumor effects in human glioblastoma cells by inhibiting IKBKE and the Akt-mTOR signaling pathway
}

\section{Jinbiao Xiong}

Tianjin Medical University General Hospital

\section{Gaochao Guo}

Tianjin Medical University General Hospital

\section{Lianmei Guo}

Tianjin Medical University General Hospital

\section{Zengguang Wang}

Tianjin Medical University General Hospital

\section{Zhijuan Chen}

Tianjin Medical University General Hospital

\section{Yang Nan}

Tianjin Medical University General Hospital

\section{Yiyao Cao}

Tianjin Medical University General Hospital

\section{Ruilong Li}

Tianjin Medical University General Hospital

\section{Xuejun Yang}

Tianjin Medical University General Hospital

\section{Jun Dong}

Second Affiliated Hospital of Soochow University

\section{Xun Jin}

Tianjin Tumor Hospital

\section{Weidong Yang}

Tianjin Medical University General Hospital

Qiang Huang ( $\sim$ haugnqiang209@163.com )

Tianjin Medical University General Hospital https://orcid.org/0000-0003-3027-1226 
Keywords: Amlexanox, Temozolomide, Glioblastoma, p-AKT, IKBKE, Chemoresistance

Posted Date: August 4th, 2020

DOI: https://doi.org/10.21203/rs.3.rs-38607/v2

License: (c) (1) This work is licensed under a Creative Commons Attribution 4.0 International License. Read Full License 


\section{Abstract}

Background: Temozolomide (TMZ), as the first-line chemotherapeutic agent for the treatment of glioblastoma multiforme (GBM), often fails to improve the prognosis of GBM patients due to the quick development of resistance. The need for more effective management of GBM is urgent. The aim of this study is to evaluate the efficacy of combined therapy with TMZ and amlexanox, a selective inhibitor of inhibitor of nuclear factor kappa-B kinase subunit epsilon (IKBKE), for GBM. Methods: in vitro, cell viability assay, apoptosis analysis, western blot, migration and invasion assay were used. In vivo, intracranial tumor models were constructed and the immunohistochemistry were used. Results: We found that combined treatment resulted in significant induction of cellular apoptosis and the inhibition of cell viability, migration and invasion in primary glioma cell and in the human glioma cell line, U87 MG. TMZ enhanced expression of phosphoration of adenosine 5'-monophosphate-activated protein kinase (pAMPK) and amlexanox led to reduction of IKBKE, with no impact on p-AMPK. Furthermore, we demonstrated that, compared to other groups treated with each component alone, TMZ combined with amlexanox effectively inhibited phosphorylation of protein kinase B (AKT) and mammalian target of rapamycin (mTOR). In addition, the combination treatment also clearly reduced in vivo tumor volume and prolonged median survival time in the xenograft mouse model. Conclusion: These results suggest that amlexanox sensitized primary glioma cell and U87 MG cell to TMZ at least partially though the suppression of IKBKE activation and the attenuation of AKT activation. Overall, combined treatment with TMZ and amlexanox may provide a promising possibility for improving the prognosis of glioblastoma patients in clinical practice.

\section{Background}

Glioblastoma multiforme (GBM), which accounts for more than $60-70 \%$ of all gliomas, is the most aggressive and most deadly primary brain tumor in adults [1,2]. Over the past decades, although there has been progress regarding human GBM treatment, currently including maximal surgical resection followed by chemotherapy and/or radiotherapy, the prognosis of patients diagnosed with GBM remains extremely grim, with a median survival of approximately 14.6 months and a 5 -year survival rate of only $9.8 \%[3,4]$. Thus, there is an overwhelming need for more efficacious therapeutic approaches for this malignancy.

Temozolomide (TMZ), a novel oral alkylating agent, is the drug that is most frequently used against malignant glioma, with broad-spectrum antitumor activity [5]. Although TMZ is considered the most promising chemotherapeutic drug against GBM, most patients suffer from tumor recurrence within 7 months due to the development of resistance [6]. Accumulating evidence demonstrates that the activation of protein kinase $B$ (AKT) is responsible for the evolution of resistance in different types of cancers [7-9]. AKT phosphorylates several substrates associated with various cellular processes, such as cell growth, survival and metabolism $[10,11]$. Interestingly, AKT activation is be enhanced by TMZ treatment, which in turn attenuates TMZ-induced apoptosis $[12,13]$. Recently, AKT is reported to be 
phosphorylated by inhibitor of nuclear factor kappa-B kinase subunit epsilon (IKBKE, also known as IKKE and IKKi) in breast cancer [14], non-small-cell cancer (NSCLC) [15], and other cells or tissues [16].

Amlexanox, a selective IKBKE inhibitor, has been approved for the treatment of aphthous ulcers and asthma without clear molecular mechanism $[17,18]$. Amlexanox is also effective for the treatment of obesity and type 2 diabetes $[19,20]$. Moreover, the potential potency of inhibition in GBM cell lines was recently demonstrated [21]. Here, we hypothesize that amlexanox can attenuate the chemoresistance of human GBM cells to TMZ by inhibiting the activation of IKBKE and AKT.

In this study, we demonstrated that the combination of $T M Z$ and amlexanox augmented the effects in primary GBM cells and human GBM cells in vitro. Furthermore, the efficacy of the combination was confirmed in vivo in a xenograft mouse model. These results suggest the possibility of TMZ combined with amlexanox for the treatment of GBM.

\section{Materials And Methods}

\section{Chemical and reagents}

Anti-IKBKE (\#3416P), anti-phospho-AKT (Ser473, \#4058S), and anti-AMPK (\#5831S) antibodies were purchased from Cell Signaling Technology, Inc (Shanghai China). Anti-mTOR (\#35373), anti-phosphomTOR (Ser2448, \#11221), and anti-caspase-3 (\#27525) antibodies were purchased from Signalway Antibody (College Park, MD, USA). Anti-AKT (\#A18675), anti-Bcl2 (\#A19693), and anti-Bax (\#A7626) antibodies were purchased from ABclonal (Boston, MA, USA). Anti-phospho-AMPK (Ser172, \# ARG51678) antibody was purchased from Arigo (Taiwan, China). Goat anti-mouse IgG-HRP, goat anti-rabbit IgG-HRP and GAPDH antibodies were purchased from Utibody (Tianjin, China). Amlexanox was purchased from Selleck.cn (Shanghai, China), and TMZ was purchased from Solarbio Science \& Technology (Shanghai, China). Both components were dissolved in dimethyl sulfoxide (DMSO) (Louis, MO, USA) to prepare a stock concentration of $100 \mathrm{mM}(\mathrm{TMZ})$ and $500 \mathrm{mM}$ (amlexanox), respectively, and stored at $-20^{\circ} \mathrm{C}$.

\section{Cell culture}

The GBM cell line (U87 MG) was kindly provided by professor Chunsheng Kang, and was maintained in Dulbecco's modified Eagle's medium (DMEM, HyClone, Logan, UT, USA) supplemented with $10 \%$ fetal bovine serum (FBS, Gibco, Thermo Fisher Scientific, Inc., Waltham, MA, USA) and $100 \mathrm{U} / \mathrm{mL}$ penicillin/streptomycin (Solarbio Science \& Technology, Co., Ltd., Shanghai, China), and cultured in a 95\% humidified atmosphere with $5 \% \mathrm{CO}_{2}$ at $37^{\circ} \mathrm{C}$.

\section{Primary human glioblastoma cells}

After obtaining informed consent, fresh tumor samples, classified as grade IV of astrocytoma based on the World Health Organization (WHO), were obtained from a patient undergoing surgical treatment at Tianjin Medical University General Hospital. Within $1 \mathrm{~h}$ of removal, a part of the resected tissues was 
washed and enzymatically digested with collagenase at $37^{\circ} \mathrm{C}$ for $1 \mathrm{~h}$. . Then, the undigested tissues were removed through centrifugation, and the rest of the sample was mixed with F12 (Gibco, Thermo Fisher Scientific, Inc.) medium supplemented with $10 \%$ FBS and $100 \mathrm{U} / \mathrm{mL}$ penicillin/streptomycin and maintained at $37^{\circ} \mathrm{C}$ in a $5 \% \mathrm{CO}_{2}$ incubator.

\section{Cell viability assay}

The Cell Counting Kit-8 (CCK-8) assay (Dojindo EU GmbH, Beijing, China) was used to evaluate cell viability. In brief, both cells (U87 MG cell and primary GBM cell) in the log growth phase were seeded in 96 -well plates at the density of $3 \times 10^{3}$ cells per well and incubated overnight in $200 \mu$ DMEM media. Then, the cells were treated with designated concentration of TMZ or amlexanox alone or both for $24,48,72 \mathrm{~h}$ and compared with negative control (NC) treated with DMSO. After incubation, $10 \mu \mathrm{l} \mathrm{CCK-8} \mathrm{was} \mathrm{added} \mathrm{to}$ each well according to the manufacturer's instructions, and the cells were incubated for $2 \mathrm{~h}$ at $37^{\circ} \mathrm{C}$. Finally, the OD value was determined at $450 \mathrm{~nm}\left(\mathrm{OD}_{450}\right)$ by a microplate reader (Synergy2, BioTek, VT, USA).

\section{Colony formation assay}

The assay included four groups: NC, TMZ, Amlexanox, and TMZ+amlexanox group. In brief, five hundred U87 MG or primary GBM cells were seeded in six-well plates. After incubation overnight, both cell types were treated with DMSO, desired concentration of TMZ and amlexanox, alone or in combination, and the medium was changed once every 5 days for 2 weeks. When cells in colony were almost more than 50 cells, methanol and crystal violet were used to fix and stain cell colonies, and colonies with more than 50 cells were counted by using an inverted microscope (Olympus, Japan). The number of colony in each group was statistically analyzed.

\section{Apoptosis assay}

As the same groups as colony formation assay, there were four groups in this assay. Annexin Vfluorescein isothiocyanate (FITC)/propidium iodide (PI) apoptosis kit (Dojindo EU GmbH, Beijing, China) was used to detected apoptosis cells. In brief, both U87 MG and primary GBM cells $\left(1-1.5 \times 10^{5}\right.$ cells/well) in the log growth phase were plated in six-well plates. After treated with DMSO, desired concentration of TMZ and amlexanox, alone or in combination, for $48 \mathrm{~h}$, both cells were harvested through trypsinization without EDTA and washed with ice-cold phosphate-buffered saline (PBS). Next, cells were re-suspended in $500 \mu \mathrm{l}$ binding solution containing $5 \mu \mathrm{l}$ annexin- $\mathrm{V}$ and $5 \mu \mathrm{l} \mathrm{Pl}$ according to manufacturer's instruction. After incubating for $15 \mathrm{~min}$ at room temperature, cells apoptosis was immediately analyzed by a FACScan flow cytometer (BD Biosciences), and further statistical analysis of apoptotic cells was conducted using FlowJo software.

\section{Western blotting}


Similarly, this assay were also divided into four groups: NC, TMZ, Amlexanox, and TMZ+amlexanox group. In brief, U87 MG and primary GBM cells were seeded in $10 \mathrm{~cm}$ dishes (Falcon) and cultured overnight. After treated with DMSO, desired concentrations of TMZ and/or amlexanox for $48 \mathrm{~h}$, cells were harvested and lysed in RIPA buffer (Beijing Solarbio Science \& Technology Co., Ltd.) supplemented with a protease inhibitor mixture (APExBIO, USA). Then cells lysed fully were collected and centrifuged at 12000 $\mathrm{r} / \mathrm{min}$ for $15 \mathrm{~min}$. Supernatants were moved into another tube, and concentrations of the total proteins were determined using the bicinchoninic acid (BCA) assay kit (Thermo Fisher Scientific, Inc.) based on the manufacturer's instructions. Equivalent amounts of proteins were separated by 6,10 or $12 \%$ SDS-PAGE gels and then transferred to polyvinylidene fluoride (PVDF) membranes (Billerica, MA, USA). After blocking in $5 \%$ skim milk at $37^{\circ} \mathrm{C}$ for $1 \mathrm{~h}$, the membranes were incubated with primary antibodies overnight at $4^{\circ} \mathrm{C}$ against rabbit anti-Bcl2 (1:1000 dilution), rabbit anti-Bax (1:1000 dilution), rabbit antiactive Casespase-3 (1:1000 dilution), rabbit anti-IKBKE (1:1000 dilution), rabbit anti-AKT (1:1000 dilution), rabbit anti-phospho-AKT (1:1000 dilution), rabbit anti-AMPK (1:1000 dilution), rabbit anti-phospho-AMPK (1:1000 dilution), rabbit anti-mTOR (1:1000 dilution), rabbit anti-pospho-mTOR (1:1000 dilution), followed by incubation with corresponding HRP-conjugated second antibodies (1:10000 dilution) for $1 \mathrm{~h}$ at room temperature. GAPDH was considered an internal reference for loading. Antigen-bound antibodies were detected using the SuperSignal West Pico Plus Chemiluminescent Substrate (Thermo Fisher Scientific, USA).

\section{Migration assay}

To assess the migration ability of U87 MG and primary GBM cells after treatment for $48 \mathrm{~h}$, scratch wound healing assay was conducted. Groups were the same as above. In brief, treated cells ( $3 \times 10^{5}$ cells/well) were seeded into six-well plates; when the cells reached $80-90 \%$ confluence in a monolayer, scratch wounds were made using a $200 \mu \mathrm{L}$ pipette tip. Then, cell debris was removed, and a microscope was used to confirm the uniform scratch width of every group. After incubation at 0,12 and $24 \mathrm{~h}$, five different fields of each well were measured and photographed using a phase-contrast microscope. In addition, Transwell filters with 8- $\mu$ m pores (Corning Costar, NY, USA) (without Matrigel) were also used to evaluate the migration ability of treated cells. The assay was conducted as described in a previous report [21].

\section{Invasion assay}

The invasion capacity of treated U87 MG and primary GBM cells was evaluated using Transwell assay with inserts of 8- $\mu \mathrm{m}$ pore size. The groups were also the same as above. In brief, after treated for $48 \mathrm{~h}$, cells were resuspended in $200 \mu \mathrm{L}$ serum-free DMEM, then seeded into the upper chamber covered with Matrigel (BD Bioscience), diluted with serum-free DMEM, and incubated for $24 \mathrm{~h}$ at $37^{\circ} \mathrm{C}$. After removing non-invading cells with cotton swabs from the top well , the bottom cells were fixed in $5 \%$ methanol, stained with $0.1 \%$ crystal violet, and then three independent $200 x$ regions were photographed randomly for each insert.

\section{Immunohistochemistry (IHC) and hematoxylin-eosin (HE) staining}


For histological analysis, the tissues were fixed in $4 \%$ formaldehyde for IHC and $\mathrm{HE}$ analysis. For IHC staining, the slides $(5 \mu \mathrm{m})$ were dewaxed using xylene and rehydrated using graded alcohols. Antigen retrieval was performed with sodium citrate $(\mathrm{pH}=6)$ buffer at $92-99^{\circ} \mathrm{C}$ for $15 \mathrm{~min}$, and then the slides were cooled at room temperature. Slides were washed three times for 5 min in PBS and incubated with 3\% $\mathrm{H}_{2} \mathrm{O}_{2}$ for 30 min to block endogenous peroxidases. The slides were blocked using $1 \%$ BSA for 30 min at room temperature. Next, slides were incubated at $4^{\circ} \mathrm{C}$ overnight with primary antibodies against rabbit anti-IKBKE (1:100 dilution), rabbit anti-phospho-AKT (1:100 dilution), rabbit anti-phospho-AMPK (1:100 dilution), rabbit anti-pospho-mTOR (1:100 dilution) before being incubated using biotin-labeled secondary antibody (1:100 dilution) for $1 \mathrm{~h}$ at $37^{\circ} \mathrm{C}$ and incubated again with diaminobenzidine (DAB) (Solarbio Science \& Technology, Beijing, China). Finally, slides were counterstained using hematoxylin and mounted. For HE analysis, slides $(\mu \mathrm{m})$ were dewaxed and rehydrated as the same as IHC staining assay. Then the nuclei were attained using hematoxylin. After rinsed in running tap water, the sections were stained with eosin. Finally, the slides were dehydrated.

\section{Xenograft models}

All mouse experiments were conducted according to protocols approved by the Tianjin Medical University Animal Care and Use Committee and followed guidelines for animal welfare. Female BALB/c-nude mice (4 weeks old, approximately $12 \mathrm{~g}$ ) were purchased from Beijing HFK Bioscience Co., Ltd. To establish an intracranial tumor model, primary GBM cells $\left(5 \times 10^{4}\right.$ cells) infected with luciferase-encoding lentivirus were stereotactically injected into the right hemisphere. A burr hole was located at a point situated $2 \mathrm{~mm}$ lateral from bregma and between bregma and fonticuli minor with a syringe under stereotactic guidance. Seven days after injection, the mice were divided randomly into 4 groups with 15 mice in each group: NC, TMZ alone, amlexanox alone and combination (TMZ and amlexanox) grorp. The NC group was treated with DMSO, and the treated groups were intraperitoneally given amlexanox alone $(100 \mathrm{mg} / \mathrm{kg}, \mathrm{TMZ}$ alone $(5 \mathrm{mg} / \mathrm{kg})$ or TMZ (5 mg/kg) and amlexanox (100 mg/kg), respectively, for 5 days. After 2 days without injections, the same dosing regimen was continuously repeated. Tumor growth was measured once every week using BLI at a designated time with the IVIS Spectrum Live Imaging System (PerkinElmer, USA). After 4 weeks post-injection, 3 mice in each group were sacrificed, and the brains were extracted and fixed in $10 \%$ formalin then embedded in paraffin for $\mathrm{HE}$ and IHC. The remaining mice were used for survival analysis.

\section{Statistical analysis:}

All experimental data are represented as the mean \pm Standard deviation (SD). Statistical analysis was performed using GraphPad Prism 6 software. One-way analysis of variance (ANOVA) was carried out to assess differences between multiple groups. The Kaplan-Meier method was used to evaluate difference in survival among the groups. $P<0.05$ was regarded as statistically significant.

\section{Results}




\section{Amlexanox enhanced the induced-TMZ suppression of GBM cell proliferation.}

To evaluate the effect of the association with TMZ and amlexanox on cell viability, the CCK-8 assay was conducted. U87 MG and primary GBM cells were treated with different doses of TMZ, amlexanox, alone or both for 24,48 , and $72 \mathrm{~h}$. As expected, the inhibition of proliferation in both cell types was gradually amplified with an increased concentration of either agent alone (Fig 1a), which was consistent with previous studies [21, 4]. Moreover, the proliferation of cells treated with the combination, whether U87 MG or primary GBM cells, was efficaciously attenuated (Fig 1a). In addition, the $48 \mathrm{~h}$ IC50 values for TMZ and amlexanox in U87 MG cells were $400 \mu \mathrm{M}$ and $300 \mu \mathrm{M}$, respectively, which were reduced to $200 \mu \mathrm{M}$ and $150 \mu \mathrm{M}$ with the combination treatment. Similarly, the IC50 value of either component alone was higher than that of the combination treatment in primary GBM cells. Based on IC50 values in our study, the concentrations of $100 \mu \mathrm{M}$ for TMZ and $50 \mu \mathrm{M}$ for amlexanox, were used in the subsequent experiments. Next, the colony formation ability of U87 MG and primary GBM cells was determined. The colony formation analysis showed that the colony formation numbers were significantly decreased in cells with the combination treatment compared to cells treated with either agent alone (Fig $\mathbf{1 b}, \mathbf{c})$.

\section{Amlexanox promoted TMZ-induced apoptosis of GBM cells.}

Apoptosis is one of the main mechanism by which TMZ acts [22, 23]. To evaluate whether amlexanox can enhance TMZ-induced apoptosis, an annexin V-FITC assay was conducted. As shown in Fig $2 \mathrm{a}$, the percentage of apoptotic cells was significantly increased in U87 MG cells with the combination treatment compared to cells treated with either drug alone, which was consistent with the findings in primary GBM cells. Furthermore, the effect of the combination on apoptosis was validated through a western blot assay in which changes in apoptosis-related proteins were induced. The activation of proapoptotic proteins, Bax and caspase-3 were higher in both cells treated with the combination than that in cells treated with either drug alone (Fig $\mathbf{2 b}$ ). The expression of antiapoptotic protein $\mathrm{Bcl}-2$ was decreased in the combination group, in both U87 MG and primary GBM cells, compared to that in cells treated with each drug alone. These results suggest the possibility that amlexanox enhanced TMZ-induced antiproliferative activity by promoting apoptosis.

\section{Amlexanox augmented the TMZ-induced inhibition of migration and invasion in GBM cells.}

Migration and invasion are the primary characteristics of GBM cells. To evaluate the effect of TMZ combined with amlexanox on the migration and invasion of U87 MG and primary GBM cells, the wound healing and transwell assays were conducted. Results of wound healing assay showed that the inhibition of cellular migration in combination group was more apparent compared to that in other groups treated with a single drug (Fig 3a,b), which was in accordance with the results of the Transwell migration assay (without Matrigel) (Fig 3c,d). For the Transwell assay (with Matrigel), the results indicated that after different treatments for $24 \mathrm{~h}$, amlexanox efficaciously augmented TMZ-induced inhibition of invasion in U87 MG and primary GBM cells (Fig $3 e, f$ ).

TMZ combined with amlexanox effectively decreased the activation of AKT. 
$T M Z$ treatment has been suggested to increase the activation of AKT, which in turn results in resistance to TMZ [12]. Moreover, the IKB kinase, IKBKE, is affirmed to be responsible for activating AKT [16, 14]. To investigate whether the amlexanox-mediated inhibition of IKBKE can attenuate the TMZ-induced activation of AKT, the alteration of relevant proteins was measured following treatment of TMZ and amlexanox, alone or both in U87 MG and primary GBM cells. The effect of TMZ or amlexanox alone on the IKBKE and AKT signaling pathways was first evaluated. After treatment with the designated dose of either agent for $48 \mathrm{~h}$ in U87 MG and primary GBM cells, results were showed as Fig 4. The western blot assay indicated that TMZ induced the activation of AKT and AMPK and decreased the phosphorylation of mTOR; the activation of IKBKE was decreased after amlexanox treatment, which was consistent with previous studies $[14,21,23]$. After treating U87 MG and primary GBM cells with both agents, the results showed that TMZ combined with amlexanox resulted in enhanced reduction of p-AKT and p-mTOR. Taking together, these results show that after treatment with TMZ alone, the slightly decreased level of $p$ mTOR may be due to TMZ-induced activation of AMPK, but AKT was activated at the same time, which may have resulted in the resistance of GBM cells to TMZ. However, amlexanox reversed the TMZmediated expression of p-AKT by inhibiting IKBKE activation, which may be part of the mechanism by which amlexanox can sensitize GBM cells to TMZ treatment.

\section{Combination of TMZ and amlexanox inhibited the growth of tumors in xenograft models.}

To evaluate the efficacy of combining TMZ and amlexanox in vivo, intracranial tumor models were constructed with primary GBM cells expressing a luciferase reporter to further validate the findings above. Results were shown as Fig 5a, b, c. The volume of intracranial tumors in the group treated with amlexanox alone or TMZ alone was modestly reduced, and the survival (24 days or 25.5 days) was slightly improved compared with the results from the control group (21 days). However, there were significant reduction of tumor burden and improvement of survival (31 days) in the combination group compared to that in amlexanox alone group or TMZ alone group. The median survival in the TMZ and amlexanox co-treatment group was longer than that in the control, amlexanox alone and TMZ alone groups. The results of HE assay also indicated that the volume of tumor in the combined group was decreased more obviously than that in other groups (Fig 5d). Moreover, the molecular mechanism was assessed by IHC staining assay (Fig $\mathbf{5 d}$ ). Results showed that, compared to treatment with control, amlexanox alone or TMZ alone, treatment of TMZ with amlexanox obviously reduced the levels of p-AKT and p-mTOR. Overall, these results suggested that amlexanox was able to penetrate the blood-brain barrier in vivo and enhance TMZ-induced inhibition of tumor growth though indirectly decreased expression of $\mathrm{p}-\mathrm{AKT}$ and $\mathrm{p}-\mathrm{mTOR}$.

\section{Discussion}

In the present study, we demonstrated that amlexanox enhanced the sensitization of GBM cells to TMZ in vitro and in vivo. As far as we know, this study was the first to use primary GBM cell, which maintained important histopathological and molecular characteristics of primary GBM tumor, to evaluate the effect of the combination of TMZ with amlexanox. 
TMZ is a common chemotherapeutic drug, and the induction of DNA adducts is primary mechanism of exerting cellular toxicity [24]. Though the administration of TMZ has improved the prognosis of GBM patients, resistance against TMZ was quickly developed [9]. The overexpression of $0^{6}$-methylguanineDNA-methyltransferase (MGMT) is one of the main resistance mechanisms for repairing $0^{6}$ methylguanine, which is an important TMZ-induced lesion resulting in breakage of DNA double-strand and subsequent apoptosis [6, 5]. In addition, immune escape after TMZ treatment[25], dysfunction of the DNA mismatch repair (MMR) system [1], as well as abnormal expression of nuclear factor erythroid 2related factor 2 (Nrf2) [26] and high expression of ATP-binding cassette (ABC) membrane transporters[2], were also demonstrated to be involved in resistance development. However, what greatly interested us is the activation of AKT, which is involved various cellular processes, such as proliferation, cell growth and survival [14]. A growing number of studies have proved that the activation of AKT is associated with resistance to TMZ treatment [27]. Moreover, administration of TMZ for GBM was proved to induce AKT activation $[12,13,4,28]$. Given that the above factors resulted in decreased TMZ-induced cellular toxicity, it is impractical to treat GBM patients with a single agent. Therefore, in this study, we conducted a series of assays to evaluate the effect of a combination of TMZ and an inhibitor, amlexanox, in the treatment of human GBM cell line and primary GBM cell.

Results of the present study showed that TMZ combined with amlexanox not only effectively inhibited proliferation, invasion and migration, which were the main challenges in the treatment of GBM, but also greatly promoted cellular apoptosis in human GBM cell line and primary GBM cell. The upregulated expression of Bax and caspase-3 validated the occurrence of apoptosis. Moreover, western blot assays suggested that TMZ treatment induced AMPK phosphorylation, which contributed to apoptosis via pmTOR inhibition [23]. To better understand the potential mechanism of amlexanox enhancing TMZinduced cellular toxicity, the relevant proteins were examined following treatment of U87 MG and primary GBM cells with either agent alone or combination. It was revealed that amlexanox treatment induced the reduction of IKBKE activation, subsequently reversed TMZ-mediated expression of $\mathrm{p}-\mathrm{AKT}$, and enhanced the suppression of $\mathrm{p}$-mTOR. These results indicated that amlexanox attenuated the chemoresistance of GBM cells to TMZ partially through amlexanox-induced inhibition of IKBKE, which resulted in the repression of TMZ-induced AKT activation. In addition, TMZ combined with amlexanox efficaciously reduced the tumor volume and improved the survival of mice in the xenograft model in vivo. To date, a growing number of studies have focused on the inhibition of the AKT signaling pathway with combined administration in GBM and other tumors [7, 29-34]. Zhiyun Yu and his colleagues suggested that TMZ combined with NVP-BEZ235 synergistically inhibited GBM cell proliferation though downregulating AKT/mTOR signaling pathway [4], which was similar to our findings. However, in our study, the primary GBM cell, which are more similar in histopathological and molecular characteristics to primary GBM tumors, were used to evaluate the effect of combination treatment. Nevertheless, it is undeniable that there are some limitations to our study. Only one kind of GBM cell line or primary GBM cell was used. If more cell lines and primary GBM cells were used, the results would be more convincing. Additionally, more assays should be conducted to further validate that the inhibition of AKT signaling pathway was induced by amlexanox, which enhanced TMZ-induced cellular toxicity. The side effect was always the 
issue that should be concerned when it comes to treatment with new combination in GBM patients. More assays should be conducted to evaluate the side effect when nude mice were managed with combination of TMZ and amlexanox in present study.

\section{Conclusion}

In conclusion, we have demonstrated that amlexanox can enhance TMZ-induced cellular toxicity; the mechanism occurs partially through that the downregulated activation of IKBKE induced by amlexanox reverses TMZ-induced activation of AKT, suggesting that the combination of TMZ and amlexanox is a possible treatment for GBM patients.

\section{Declarations}

\section{Ethics approval and consent to participate}

The tissues used in the present study were approved by the Ethics Committee of Tianjin Medical University General Hospital and written informed consent was obtained (IRB2019-WZ-142).

All procedures performed in studies involving animals were in accordance with the ethical standards of the Animal Care and Use Committee of Tianjin Medical University at which the studies were conducted

\section{Consent for publication}

Not application.

\section{Availability of data and materials}

Not application.

\section{Conflict interests}

The authors declare that they have no conflict of interest.

\section{Funding:}

This study was funded by the National Natural Science Foundation of China (Grant N0.81572490, N0.81172405), Tianjin Science and Technology Committee (Grant NO.18JCZDJC98600).

\section{Author ' contributions}

Jinbiao Xiong, Gaochao Guo and Lianmei Guo have made substantial contributions to conception and design; Ruilong Li and Xuejun Yang and Xun Jin help with acquisition of data, analysis and interpretation of data; Zhijuan Chen, Yang Nan, Jun Dong and Yiyao Cao provided technical help, writing assistance; Jinbiao Xiong and Zengguang Wang were involved in drafting the manuscript and revising it critically for 
important intellectual content; Qiang Huang and Weidong Yang gave final approval of the version to be published and agreed to be accountable for all aspects of the work in ensuring that questions related to the accuracy or integrity of any part of the work are appropriately investigated and resolved

\section{Acknowledgements}

Not application.

\section{References}

1. Sun Q, Pei C, Li Q, Dong T, Dong Y, Xing W et al. Up-regulation of MSH6 is associated with temozolomide resistance in human glioblastoma. Biochem Biophys Res Commun. 2018;496(4):1040-6. doi:10.1016/j.bbrc.2018.01.093.

2. Uribe D, Torres A, Rocha JD, Niechi I, Oyarzun C, Sobrevia L et al. Multidrug resistance in glioblastoma stem-like cells: Role of the hypoxic microenvironment and adenosine signaling. Mol Aspects Med. 2017;55:140-51. doi:10.1016/j.mam.2017.01.009.

3. Guan $\mathrm{H}$, Zhang H, Cai J, Wu J, Yuan J, Li J et al. IKBKE is over-expressed in glioma and contributes to resistance of glioma cells to apoptosis via activating NF-kappaB. J Pathol. 2011;223(3):436-45. doi:10.1002/path.2815.

4. Yu Z, Xie G, Zhou G, Cheng Y, Zhang G, Yao G et al. NVP-BEZ235, a novel dual PI3K-mTOR inhibitor displays anti-glioma activity and reduces chemoresistance to temozolomide in human glioma cells. Cancer Lett. 2015;367(1):58-68. doi:10.1016/j.canlet.2015.07.007.

5. Qian Z, Zhou S, Zhou Z, Yang X, Que S, Lan J et al. miR146b5p suppresses glioblastoma cell resistance to temozolomide through targeting TRAF6. Oncol Rep. 2017;38(5):2941-50. doi:10.3892/or.2017.5970.

6. Stritzelberger J, Distel L, Buslei R, Fietkau R, Putz F. Acquired temozolomide resistance in human glioblastoma cell line U251 is caused by mismatch repair deficiency and can be overcome by Iomustine. Clin Transl Oncol. 2018;20(4):508-16. doi:10.1007/s12094-017-1743-x.

7. Zou W, Ma X, Hua W, Chen B, Cai G. Caveolin-1 mediates chemoresistance in cisplatin-resistant ovarian cancer cells by targeting apoptosis through the Notch-1/Akt/NF-kappaB pathway. Oncol Rep. 2015;34(6):3256-63. doi:10.3892/or.2015.4320.

8. Liu J-F, Tsao Y-T, Hou C-H. Fractalkine CX3CL1 induced intercellular adhesion molecule-1-dependent tumor metastasis through the CX3CR1 PI3K Akt NF-KB pathway in human osteosarcoma. Oncotarget. 2017;8:54136-48.

9. Yang WB, Chuang JY, Ko CY, Chang WC, Hsu TI. Dehydroepiandrosterone Induces Temozolomide Resistance Through Modulating Phosphorylation and Acetylation of Sp1 in Glioblastoma. Mol Neurobiol. 2018. doi:10.1007/s12035-018-1221-7.

10. Molina JR, Hayashi Y, Stephens C, Georgescu M-M. Invasive Glioblastoma Cells Acquire Stemness and Increased Akt Activation. Neoplasia. 2010;12(6):453-IN5. doi:10.1593/neo.10126. 
11. Manning BD, Cantley LC. AKT/PKB signaling: navigating downstream. Cell. 2007;129(7):1261-74. doi:10.1016/j.cell.2007.06.009.

12. Caporali S, Levati L, Starace G, Ragone G, Bonmassar E, Alvino E et al. AKT is activated in an ataxiatelangiectasia and Rad3-related-dependent manner in response to temozolomide and confers protection against drug-induced cell growth inhibition. Mol Pharmacol. 2008;74(1):173-83. doi:10.1124/mol.107.044743.

13. Hirose Y, Katayama M, Mirzoeva OK, Berger MS, Pieper RO. Akt Activation Suppresses Chk2Mediated, Methylating Agent-Induced G2 Arrest and Protects from Temozolomide-Induced Mitotic Catastrophe and Cellular Senescence. Cancer Res. 2005.

14. Guo JP, Coppola D, Cheng JQ. IKBKE protein activates Akt independent of phosphatidylinositol 3kinase/PDK1/mTORC2 and the pleckstrin homology domain to sustain malignant transformation. $J$ Biol Chem. 2011;286(43):37389-98. doi:10.1074/jbc.M111.287433.

15. Challa S, Guo JP, Ding X, Xu CX, Li Y, Kim D et al. IKBKE Is a Substrate of EGFR and a Therapeutic Target in Non-Small Cell Lung Cancer with Activating Mutations of EGFR. Cancer Res. 2016;76(15):4418-29. doi:10.1158/0008-5472.CAN-16-0069.

16. Xie X, Zhang D, Zhao B, Lu MK, You M, Condorelli G et al. IkappaB kinase epsilon and TANK-binding kinase 1 activate AKT by direct phosphorylation. Proc Natl Acad Sci U S A. 2011;108(16):6474-9. doi:10.1073/pnas.1016132108.

17. Bell J. Amlexanox for the treatment of recurrent aphthous ulcers. Clin Drug Invest. 2005;25 (9):55566.

18. Makino H, Saijo T, Ashida Y, Kuriki H, Maki Y. Mechanism of action of an antiallergic agent, amlexanox (AA-673), in inhibiting histamine release from mast cells. Acceleration of cAMP generation and inhibition of phosphodiesterase. International archives of allergy and applied immunology. 1987;82(1):66-71. doi:10.1159/000234292.

19. Beyett TS, Gan X, Reilly SM, Chang L, Gomez AV, Saltiel AR et al. Carboxylic Acid Derivatives of Amlexanox Display Enhanced Potency toward TBK1 and IKKepsilon and Reveal Mechanisms for Selective Inhibition. Mol Pharmacol. 2018;94(4):1210-9. doi:10.1124/mol.118.112185.

20. Oral EA, Reilly SM, Gomez AV, Meral R, Butz L, Ajluni N et al. Inhibition of IKKvarepsilon and TBK1 Improves Glucose Control in a Subset of Patients with Type 2 Diabetes. Cell Metab. 2017;26(1):15770 e7. doi:10.1016/j.cmet.2017.06.006.

21. Liu Y, Lu J, Zhang Z, Zhu L, Dong S, Guo G et al. Amlexanox, a selective inhibitor of IKBKE, generates anti-tumoral effects by disrupting the Hippo pathway in human glioblastoma cell lines. Cell Death Dis. 2017;8(8):e3022. doi:10.1038/cddis.2017.396.

22. De Salvo M, Maresca G, D'Agnano I, Marchese R, Stigliano A, Gagliassi R et al. Temozolomide induced c-Myc-mediated apoptosis via Akt signalling in MGMT expressing glioblastoma cells. Int J Radiat Biol. 2011;87(5):518-33. doi:10.3109/09553002.2011.556173.

23. Zhang WB, Wang Z, Shu F, Jin YH, Liu HY, Wang QJ et al. Activation of AMP-activated protein kinase by temozolomide contributes to apoptosis in glioblastoma cells via p53 activation and mTORC1 
inhibition. J Biol Chem. 2010;285(52):40461-71. doi:10.1074/jbc.M110.164046.

24. Drablos F, Feyzi E, Aas PA, Vaagbo CB, Kavli B, Bratlie MS et al. Alkylation damage in DNA and RNArepair mechanisms and medical significance. DNA Repair (Amst). 2004;3(11):1389-407. doi:10.1016/j.dnarep.2004.05.004.

25. Wang S, Yao F, Lu X, Li Q, Su Z, Lee J-H et al. Temozolomide promotes immune escape of GBM cells via upregulating PD-L1. Am J Cancer Res. 2019;9(6):1161-71.

26. Zhang L, Wang H. FTY720 inhibits the Nrf2/ARE pathway in human glioblastoma cell lines and sensitizes glioblastoma cells to temozolomide. Pharmacol Rep. 2017;69(6):1186-93. doi:10.1016/j.pharep.2017.07.003.

27. Yi GZ, Liu YW, Xiang W, Wang H, Chen ZY, Xie SD et al. Akt and beta-catenin contribute to TMZ resistance and EMT of MGMT negative malignant glioma cell line. J Neurol Sci. 2016;367:101-6. doi:10.1016/j.jns.2016.05.054.

28. Caporali S, Levati L, Graziani G, Muzi A, Atzori MG, Bonmassar E et al. NF-kB is activated in response to temozolomide in an AKT-dependent manner and confers protection against the growth suppressive effect of the drug. J Transl Med. 2012;10(252).

29. Bi Y, Li H, Yi D, Sun Y, Bai Y, Zhong S et al. Cordycepin Augments the Chemosensitivity of Human Glioma Cells to Temozolomide by Activating AMPK and Inhibiting the AKT Signaling Pathway. Mol Pharm. 2018;15(11):4912-25. doi:10.1021/acs.molpharmaceut.8b00551.

30. Wu Y, Dong L, Bao S, Wang M, Yun Y, Zhu R. FK228 augmented temozolomide sensitivity in human glioma cells by blocking PI3K/AKT/mTOR signal pathways. Biomed Pharmacother. 2016;84:462-9. doi:10.1016/j.biopha.2016.09.051.

31. Lee JE, Lim JH, Hong YK, Yang SH. High-Dose Metformin Plus Temozolomide Shows Increased Antitumor Effects in Glioblastoma In Vitro and In Vivo Compared with Monotherapy. Cancer research and treatment : official journal of Korean Cancer Association. 2018;50(4):1331-42. doi:10.4143/crt.2017.466.

32. Chen L, Han L, Shi Z, Zhang K, Liu Y, Zheng Y et al. LY294002 enhances cytotoxicity of temozolomide in glioma by down-regulation of the PI3K/Akt pathway. Mol Med Rep. 2012;5(2):575-9. doi:10.3892/mmr.2011.674.

33. Zhou W, Wang J, Qi Q, Feng Z, Huang B, Chen A et al. Matrine induces senescence of human glioblastoma cells through suppression of the IGF1/PI3K/AKT/p27 signaling pathway. Cancer Med. 2018;7(9):4729-43. doi:10.1002/cam4.1720.

34. Dai C, Zhang B, Liu X, Ma S, Yang Y, Yao Y et al. Inhibition of PI3K/AKT/mTOR pathway enhances temozolomide-induced cytotoxicity in pituitary adenoma cell lines in vitro and xenografted pituitary adenoma in female nude mice. Endocrinology. 2013;154(3):1247-59. doi:10.1210/en.2012-1908.

\section{Figures}



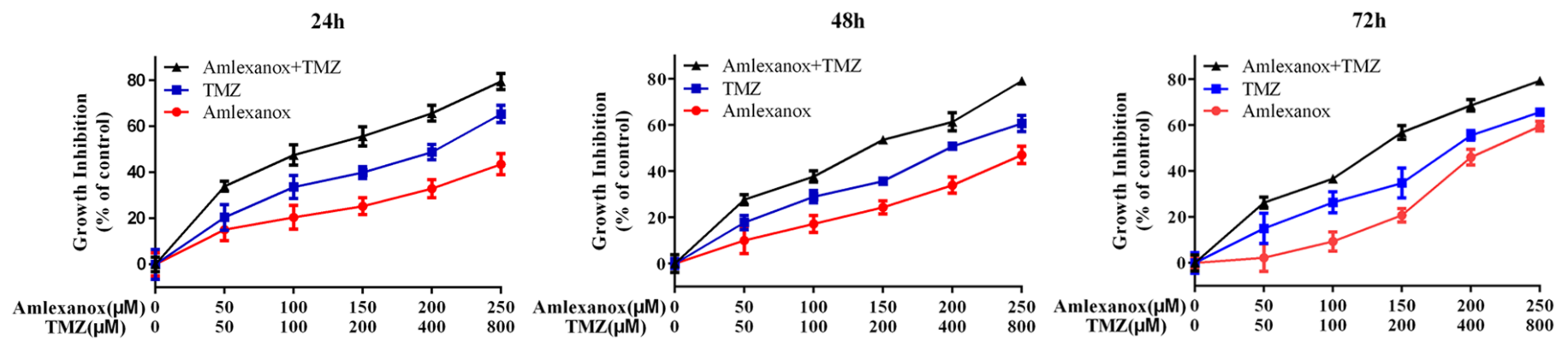

Primary GBM Cell
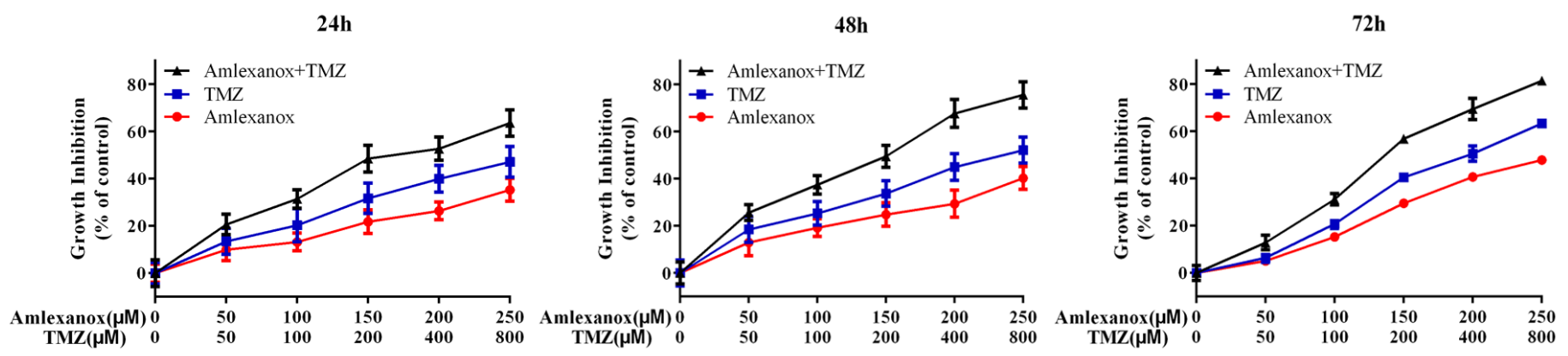

b

Amlexanox

U87 MG

c

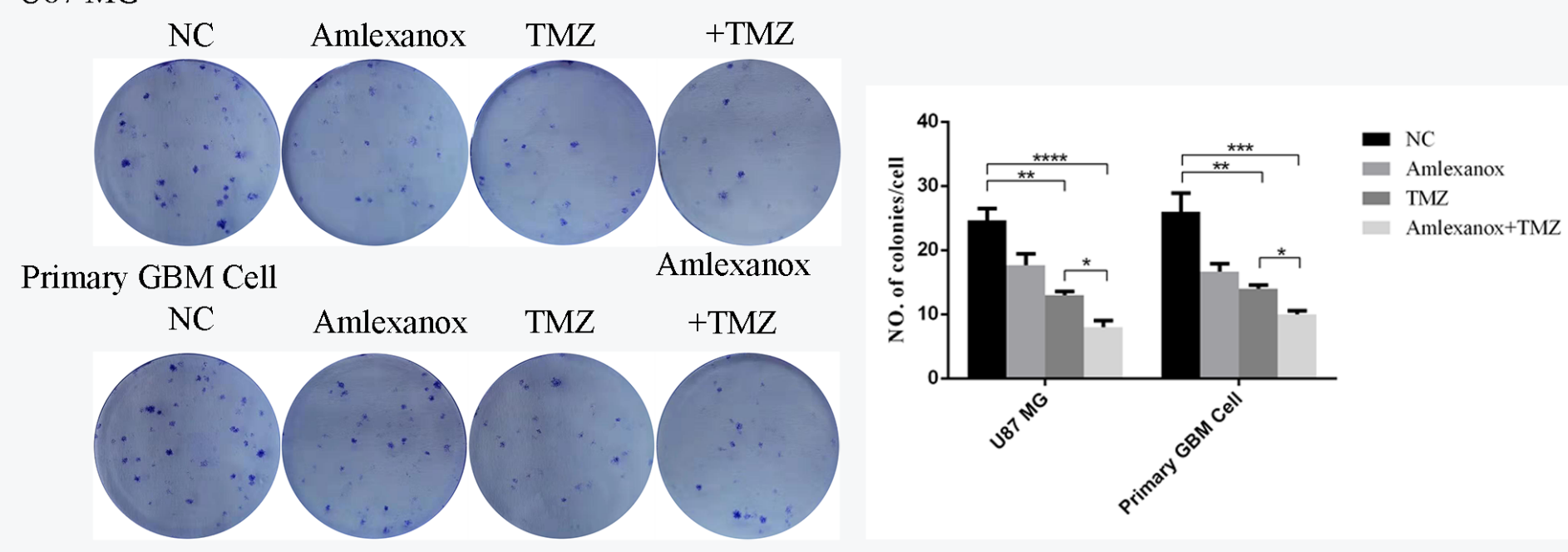

Figure 1

TMZ combined with amlexanox effectively inhibited proliferation of U87 MG and primary GBM cells. (a) The results of CCK-8 assay. U87 MG were treated with TMZ in the concentration of $0,50,100,200,400$, $800 \mu \mathrm{M}$, and primary GBM cells were treated with amlexanox in the concentration of $0,50,100,150,200$, $250 \mu \mathrm{M}$. (b, c) Representative images of the colony forming assay (left) and statistical analysis in the form of histogram (right) after exposure to TMZ $(100 \mu \mathrm{M})$, amlexanox $(50 \mu \mathrm{M})$, or both for two weeks. 
The data presented are shown as the means \pm SED. Three experiments were performed independently. ( ${ }^{*} p$ $<0.05, * * p<0.01, * * * p<0.005$ )

a

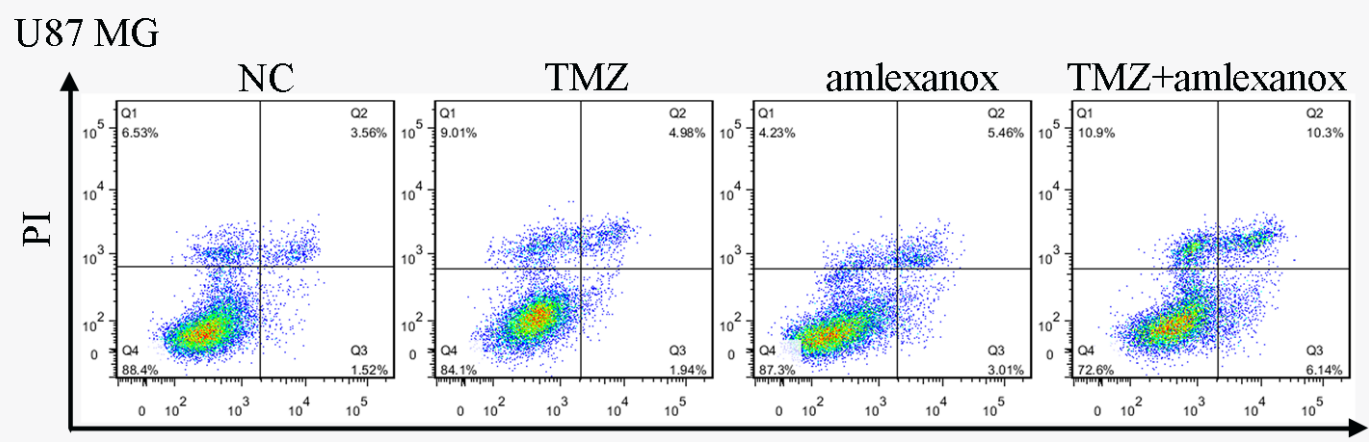

Annexin V-FITC

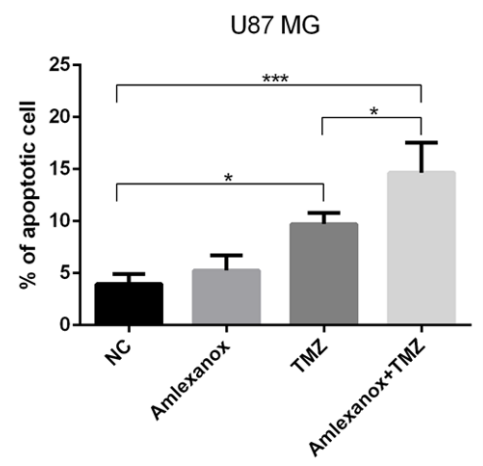

Primary GBM Cell
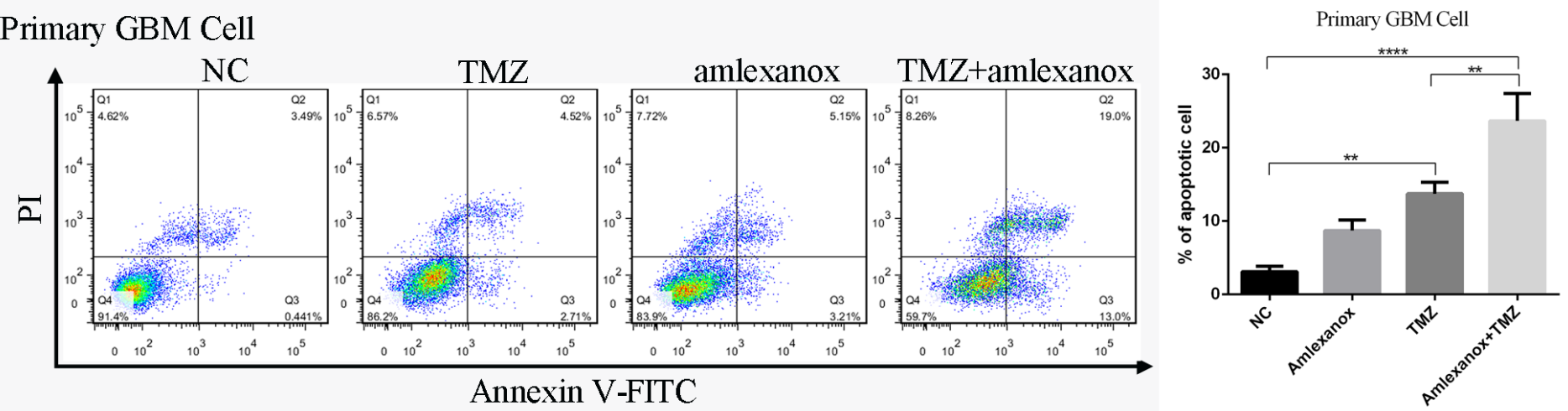

b
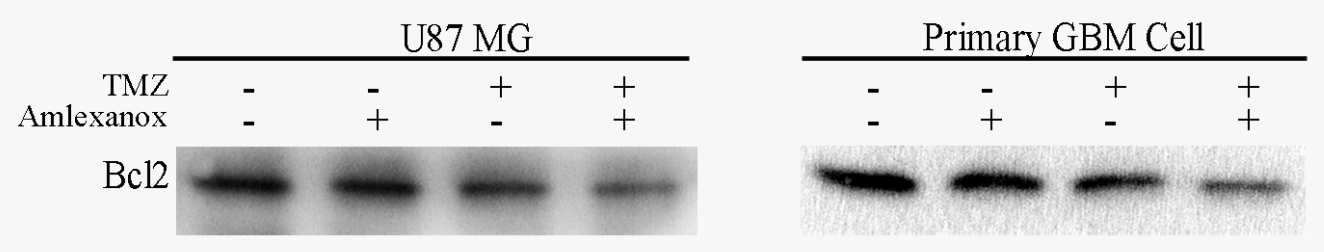

Bax
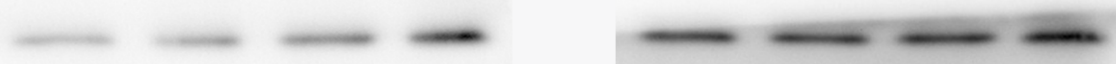

Caspase-3

GAPDH

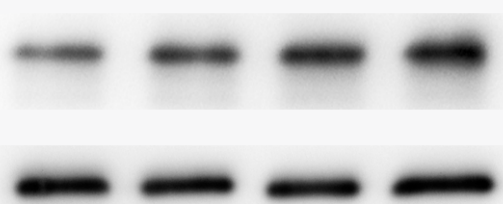

\section{Figure 2}

Amlexanox prompted TMZ-induced apoptosis in U87 MG and primary GBM cells. (a) Representative images of apoptosis (left) and quantified results in histograms (right) for U87 MG and primary GBM cells after treatment with $\mathrm{TMZ}(100 \mu \mathrm{M})$, amlexanox $(50 \mu \mathrm{M})$, or combination for $72 \mathrm{~h}$. (b) The levels of expression of $\mathrm{Bcl} 2$, Bax and active caspase-3 were measured by western blotting. The data presented 
here are shown as the mean \pm SED. Three experiments were performed independently. $\left(* P<0.05,{ }^{*} \mathrm{p}<\right.$ $0.01, * * * p<0.005$, and $* * * * p<0.001$ )

a

${ }^{\mathrm{U}} 87 \mathrm{MG}$ NC
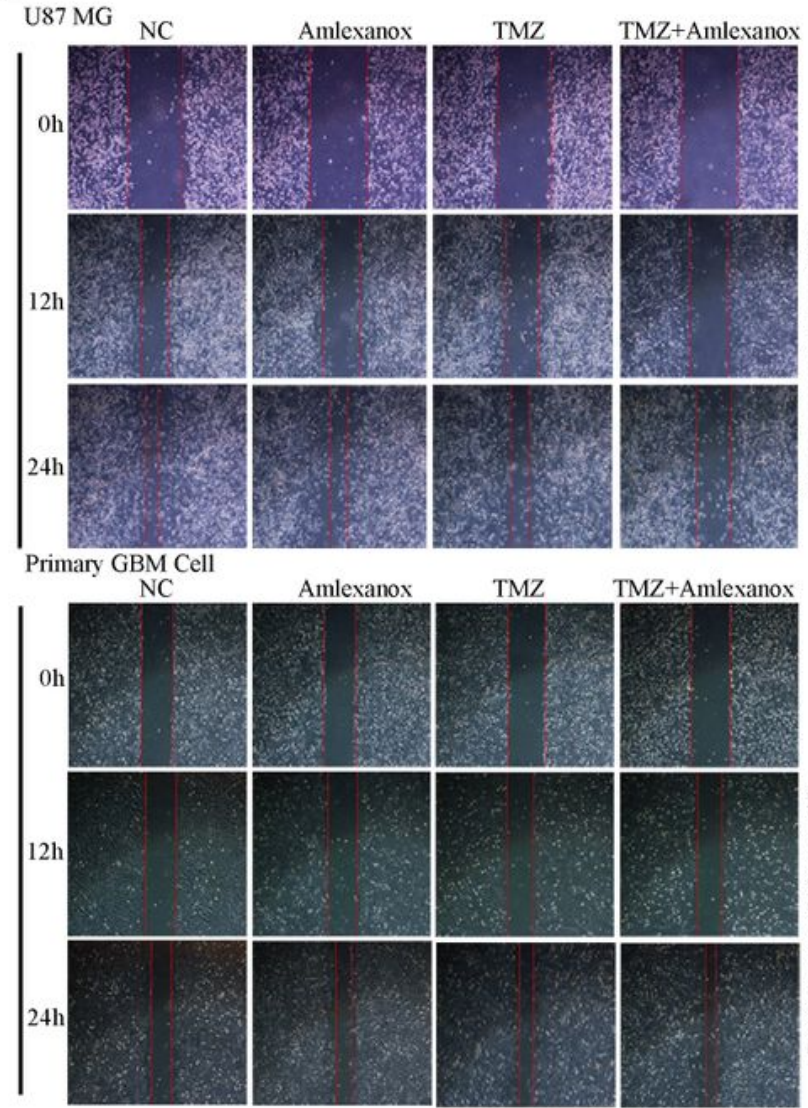

c U87 MG

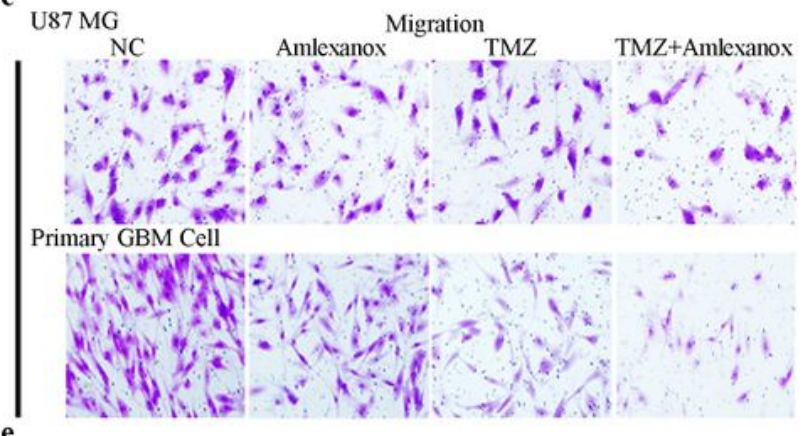

e $\mathrm{U} 87 \mathrm{MG}$

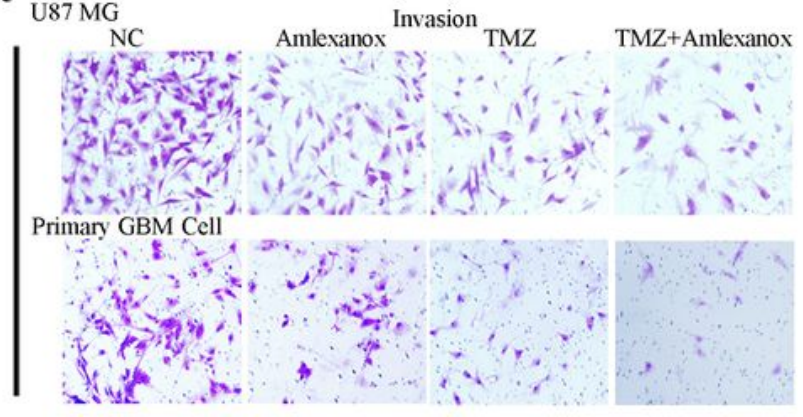

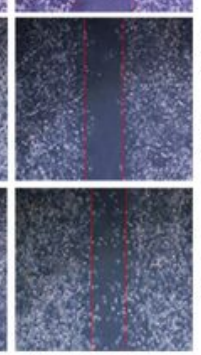

b

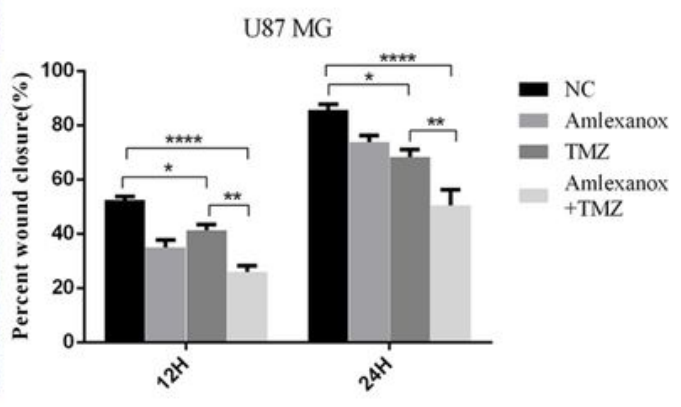

TMZ+Amlexanox
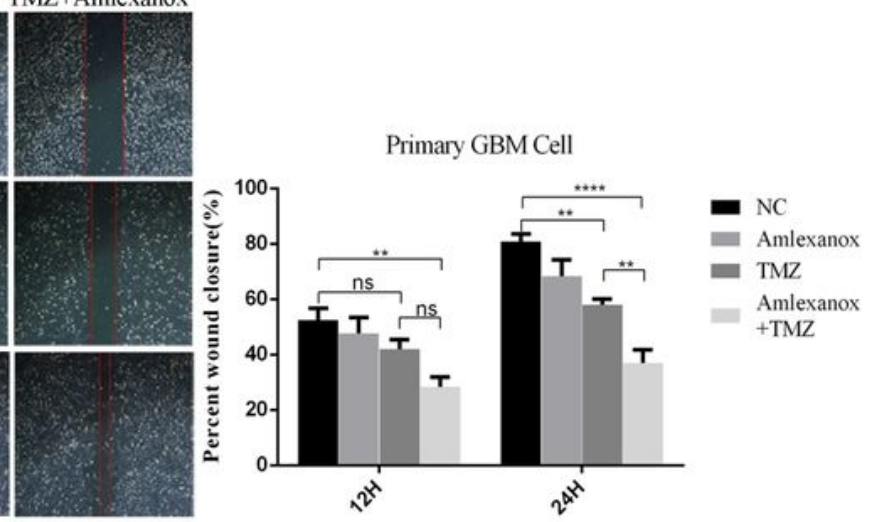

d

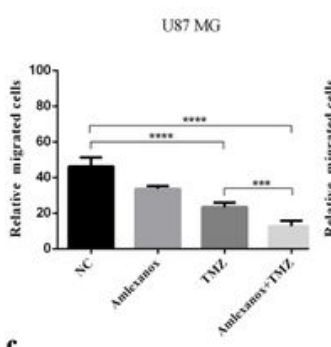

f

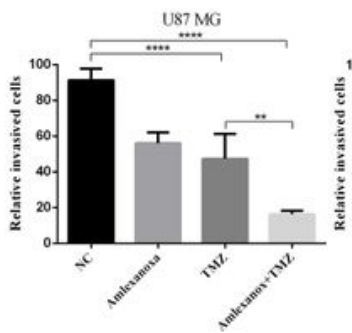

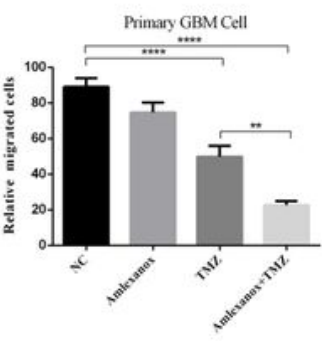

Primary GBM Cell

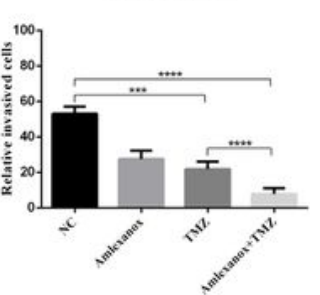

Figure 3

Combined treatment of TMZ with amlexanox efficaciously inhibited the migration and invasion of U87 MG and primary GBM cells. (a) Representative images of the wound healing assay for each cell type after 0,12 and $24 \mathrm{~h}$ seeding. (b) The data of the wound healing assay were quantified and shown as a 
histogram. (c) Representative images of both cell types were captured after 24 seeding in the Transwell assay (without Matrigel). (d) The results of both cell types from the Transwell assay (without Matrigel) were quantified and are shown in a histogram. (e) Representative images of both cell types were captured after 24 seeding in the Transwell assay (with Matrigel). (f) The results of both cell types from the Transwell assay (with Matrigel) were quantified and are shown in a histogram. Three experiments were performed independently. $(* * p<0.01$, $* * * p<0.005$, and $* * * * p<0.001)$

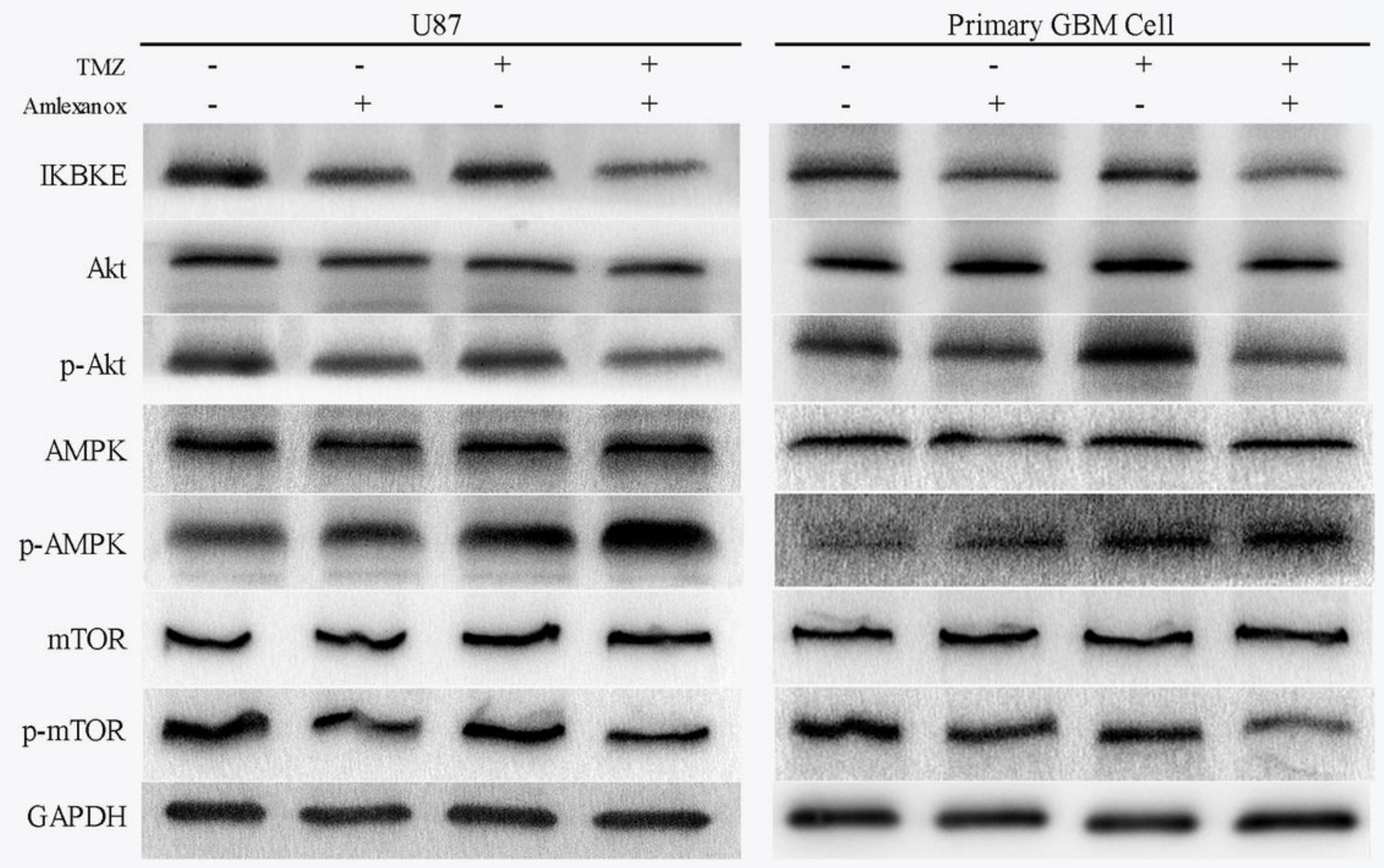

\section{Figure 4}

Amlexanox sensitized U87 MG and primary GBM cells to TMZ partially through inhibition of AKT activation. After treatment with TMZ and/or amlexanox for $48 \mathrm{~h}$, the cells were harvested, and western blotting was conducted to detect the expression of relevant proteins. 


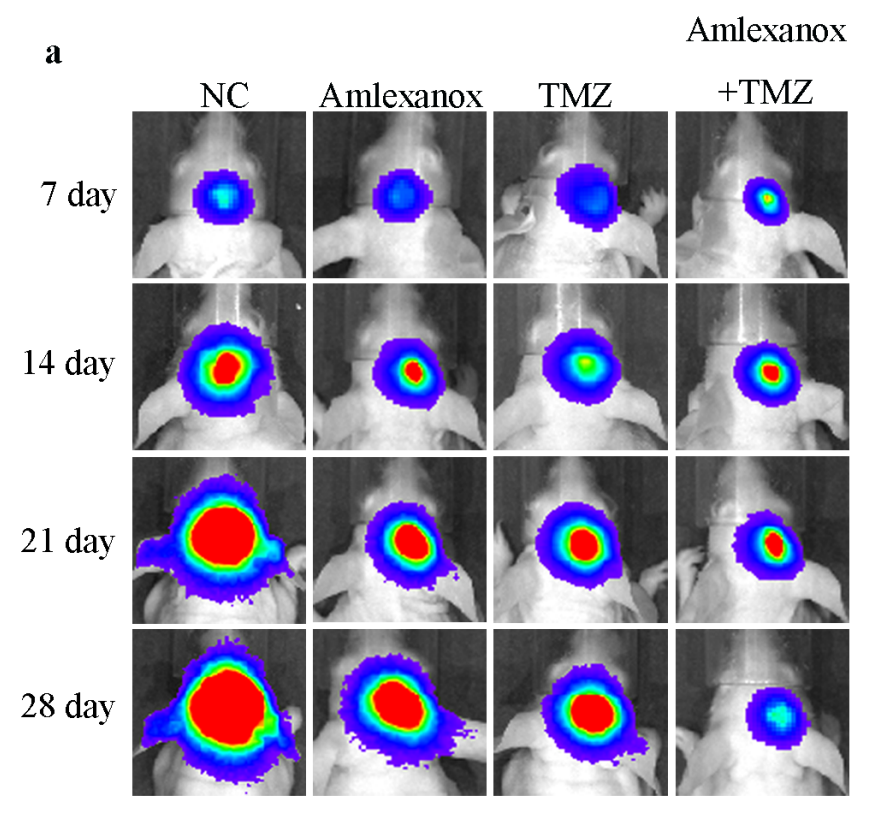

b

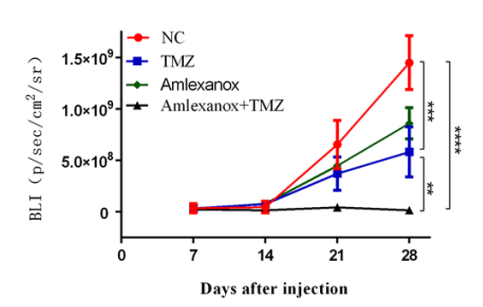

c

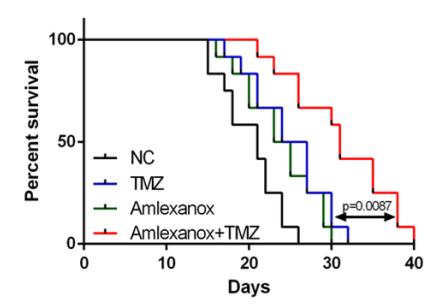

d

Amlexanox

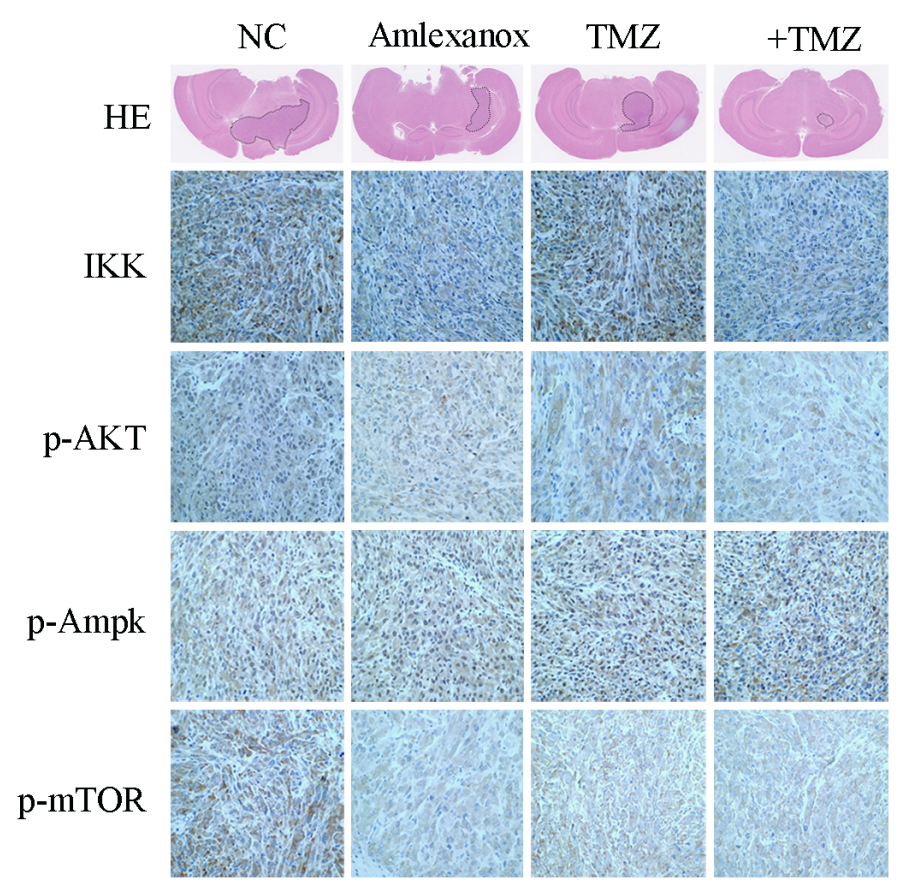

\section{Figure 5}

Amlexanox treatment sensitized orthotopic intracranial tumors of primary GBM cell to TMZ. (a) Representative images of the bioluminescence (BLI) of intracranial tumor models were captured on days 7, 14, 21, and 28. (b) Quantified analysis of these bioluminescence images for each group. (c) Survival analysis of the mice in each group. (d) Representative images of HE staining of full-brain sections and 
representative images of the IHC staining assay for IKBKE, p-AKT, p-mTOR, and p-AMPK. $(\times 200$ magnification) ( ${ }^{\star \star} p<0.01, * \star * p<0.005$, and $\left.* * \star * p<0.001\right)$ 